\title{
Methodology of Epidemic Risk Management in Kazakhstan with Open-Source EIDSS
}

\author{
Aizhan Esmagambetova ${ }^{2}$, Alexey Burdakov*1, Stanislav Kazakov ${ }^{3}$, Andrey Ukharov ${ }^{1}$, Erlan \\ Sansyzbaev $^{3}$ and Vladimir Kazakov ${ }^{3}$
}

${ }^{1}$ Black \& Veatch, Overland Park, KS, USA; '2Agency of the Republic of Kazakhstan on Consumer Rights Protection, Astana, Kazakhstan; ${ }^{3}$ Kazakh Scientific Center of Quarantine and Zoonotic Diseases, Almaty, Kazakhstan

\section{Objective}

Development and approbation of the epidemic risk estimation and management methodology based on multivariate analysis per administrative clusters of Kazakhstan using the Electronic Integrated Disease Surveillance System (EIDSS) technical capabilities.

\section{Introduction}

In order to transition the forecasting, estimation and management of epidemic risks to individual administrative areas, the Agency for Consumer Rights Protection of Kazakhstan has developed a concept for modernizing the existing national system of the epidemiological surveillance (SES).

It is proposed that the data from the SES (epidemiology, sanitary and epidemiological background, external environmental objects and database) will be consolidated to generate a new epidemic risk control and management tool called the Regional Sanitary-Epidemiological Passport (RSEP) for each of Kazakhstan's districts. The RSEP will contain infectious incidence rate dynamics according to the primary (marker) infections (7 nosologies) including a forecast for 2-3 years, and natural and soil foci GIS maps for especially dangerous pathogens (EDP) with a 3-5 year forecast of their activity.

The RSEP is planned as a new working tool for epidemiologists to aid in making objective estimates, forecasting epidemic risks in particular areas of Kazakhstan, and taking preventive steps to lower epidemic risks.

\section{Methods}

The RSEP methodology is based on area specific data of infectious incidence rate marker indicators, and the environmental conditions based on sanitary-epidemiological and socio-economic spheres. Basic nosological forms registered in all regions and determining the overall infectious morbidity are used as the epidemic process markers for districts. The average long-term morbidity data per 100,000 of population for a period of $10+$ years is used to determine the marker levels permissible (typical) for a particular area.

An auxiliary forecast component is the immune background for the main vaccines in use, including BCG vaccination of children up to 1 year of age in maternity hospital.

EDP natural and soil foci and their activity of epizootic manifestations determine the risk level for populations living in unfavorable areas. Since population sporadic morbidity in these foci hinders the ability to forecast morbidity, RSEP also contains GIS maps of natural (soil) foci both basic and significant for most of the areas, and their distribution and epizootic activity based on long-term observations.

Public catering facilities, schools and food industry facilities have the greatest impact on the markers of the epidemic process selected in RSEP.

Based on a set of risk assessment sanitary-epidemiological indicators, an aggregate report is generated, which describes the situation in the region with the control criteria for the markers.
The open-source EIDSS will serve as the main tool of the proposed methodology in addition to its functions of collecting, processing and analyzing epidemiological, clinical and laboratory information on 64 controlled infectious diseases in 269 institutions of the Agency as the national SES.

In 2012-13 we tested the EIDSS capability to forecast the disease risk for the Kazakhstani population of contracting the Crimean-Congo hemorrhagic fever. The forecast provided by the application proved to be $81.3 / 88.9 \%$ accurate [1].

\section{Results}

The concept and template methodology of estimation and management of epidemic risks have been developed. Its approbation by the CCHF has been conducted, and a high level of accuracy was shown [1]. Tasks for the detailed design and implementation of the methodology were formulated, including:

- Adjustment of estimation methods (increasing of the number of factors, use of specific algorithms for individual nosologies)

- Formation of a reach back data bank 15-20 years deep on 7 infections based on historical data

- Creation of an electronic archive of GIS maps of EDP natural foci

- Application of EIDSS for implementation of a substantial portion or the whole methodology.

\section{Conclusions}

The methodology approbation results (CCHF) and availability of the EIDSS electronic system at the disposal of the Agency allow us to conclude the efficiency of the methodology and, upon its further development, to introduce it as a tool available to epidemiologists from national to district levels for effective risk assessment and management.

\section{Keywords}

risk management; EIDSS; marker indicators; surveillance system

\section{References}

1. Esmagambetova A., et.al. Accuracy of EIDSS software prognosis on CCHF natural foci activity in Kazakhstan. OJHPI. 2014; 6(1).

\author{
*Alexey Burdakov \\ E-mail: burdakov@usa.net
}

